\title{
On a heat kernel for the index Whittaker transform
}

\section{M. RODRIGUES and S. YAKUBOVICH}

\section{ABSTRACT.}

We introduce a notion of the heat kernel associated with the index Whittaker transform. We study its differential and mapping properties and a relationship with a family of the corresponding Weierstrass's type transforms.

Acknowledgements. The work of the first author was supported in part by FEDER funds through COMPETE- Operational Programme Factors of Competitiveness ("Programa Operacional Factores de Competitividade") and by Portuguese funds through the Center for Research and Development in Mathematics and Applications and the Portuguese Foundation for Science and Technology ("FCT-Fundao para a Cincia e a Tecnologia"), within project PEst-C/MAT/UI4106/2011 with COMPETE number FCOMP-01-0124-FEDER-022690.

The work of the second author was supported by the European Regional Development Fund through the programme COMPETE and by the Portuguese Government through the FCT Fundação para a Ciência e a Tecnologia under the project PEst-C/MAT/UI0144/2011.

\section{REFERENCES}

[1] Becker, P. A., On the integration of products of Whittaker functions with respect to the second index, J. Math. Phys., 45 (2004), 761-773

[2] Buchholz, H., The Confluent Hypergeometric Function with Special Emphasis on Its Applications, Springer, Berlin, 1969

[3] Erdélyi, A., Magnus, W., Oberhettinger, F. and Tricomi, F. G., Higher Transcendental Functions-Vol. I, II, McGraw-Hill, New York, London and Toronto, 1953

[4] Prudnikov, A. P., Brychkov, Yu. A. and Marichev, O. I., Integrals and Series-Vol. 1: Elementary Functions, Gordon and Breach, 1986

[5] Prudnikov, A. P., Brychkov, Yu. A. and Marichev, O. I., Integrals and Series-Vol. 2: Special Functions, Gordon and Breach, 1986

[6] Prudnikov, A. P., Brychkov, Yu. A. and Marichev, O. I., Integrals and Series-Vol. 3: More Special Functions, Gordon and Breach, 1989

[7] Sneddon, I. N.,The Use of Integral Transforms, McGraW-Hill, New York, 1972

[8] Srivastava, H. M., Vasil'ev, Yu. V. and Yakubovich, S., A class of index transforms with Whittaker's function as the kernel, Quart. J. Math. Oxford, Quart. J. Math. Oxford Ser. (2), 49 (1998), No. 195, 375-394

[9] Szmytkowski, R. and Bielski, S., An orthogonality relation for the Whittaker functions of the second kind of imaginary order, Integral Transforms Spec. Funct., 21 (2010), 739-744

[10] Wimp, J., A class of integral transforms, Proc. Edinburgh Math. Soc., 14 (1964), No. 2, 33-40

[11] Yakubovich, S., The Kontorovich-Lebedev transformation on Sobolev type spaces, Sarajevo J. of Mathematics, 1 (2005), No. 14, 211-234

[12] Yakubovich, S., The heat kernel and Heisenberg inequalities related to the Kontorovich-Lebedev transform, Commun. Pure. Appl. Anal., 10 (2011), No. 2, 745-760

Received: 16.04.2012; In revised form: 02.10.2012; Accepted: 22.12 .2012

2010 Mathematics Subject Classification. 44A15, 44A05, 44A35.

Key words and phrases. Index Whittaker transform, Whittaker function, modified Bessel function, heat kernel.

Corresponding author: M. M. Rodrigues; mrodrigues@ua.pt 
[13] Yakubovich, S., Index Transforms, World Scientific Publishing Company, Singapore, New Jersey, London and Hong Kong, 1996

[14] Yakubovich, S., and Luchko, Yu. F., The Hypergeometric Approach to Integral Transforms and Convolutions, (Kluwers Ser. Math. and Appl., Vol. 287), Dordrecht, Boston, London, 1994

CIDMA - CENTER For ResearCh AND DEVElopment in MATHEMATICS AND APplications DEPARTMENT OF MATHEMATICS, UNIVERSITY OF AVEIRO

Campus Universitário de Santiago, 3810-193 Aveiro, Portugal

E-mail address: mrodrigues@ua.pt

Department of Mathematics

UNIVERSITY OF PORTO

FACULDADE DE CIÊNCIAS

Rua do Campo Alegre 687, 4169-007 Porto, Portugal

E-mail address: syakubov@fc.up.pt 